PII: S0960-0779(98)00129-X

\title{
Nonlinear Non-Relativistic Gravity
}

\author{
ROMAN TOMASCHITZ \\ Department of Physics, Hiroshima University, 1-3-1 Kagami-yama, Higashi-Hiroshima, 724, Japan \\ Indian Institute of Astrophysics, Koramangala, Bangalore 560 034, India
}

(Accepted 18 June 1997)

\begin{abstract}
A phenomenological theory of gravitation is proposed, based on a pre-relativistic approach to electromagnetism. The general relativistic, purely geometric, spacetime conception is criticized. Space itself is assumed to have physical substance. This substance, the ether, manifests by its permeability. Gravitational fields do not curve space, but rather distort the ether, and so affect its permeability. The permeability determines the speed of light, which is varying as in a dielectric medium. The theory is only in certain limits Lorentz invariant; the concept of a uniformly moving observer is likewise only approximate, and so is the principle of equivalence. Nonlinear field equations for the scalar gravitational field as well as the permeability tensor of the ether are constructed in a way to fit the classical experiments on gravitation. Estimates on the variation of the speed of light in our solar system are given. (C) 1998 Elsevier Science Ltd. All rights reserved
\end{abstract}

\section{INTRODUCTION}

Einstein's principle of special relativity stands out in the physics of the twentieth century as the starting point of a new way of thinking about space and time. It led, at first purely formally, to the introduction of time as a dimension in a 4-dimensional pseudo-Euclidean space, endowed with an indefinite metric $\mathrm{d} s^{2}=-c^{2} \mathrm{~d} t^{2}+\mathrm{d} \mathbf{x}^{2}$. To view space and time as a 4-manifold, one needs a universal constant of the dimension of velocity to equalize the space and time intervals $\mathrm{d} t$ and $\mathrm{d} \mathbf{x}$ dimensionally. This constant $c$ enters in the theory of special relativity as the vacuum speed of light. The coordinate frames of uniformly moving observers are related by Lorentz transformations, which leave $\mathrm{d} s^{2}$ invariant. The introduction of a 4-dimensional spacetime world turns out to be very useful and suggestive. The requirement of manifest covariance (forminvariance of evolution equations under Lorentz transformations) provides a very strong selection criterion for possible interactions in relativistic quantum systems.

However, in the context of special relativity, the introduction of time as a dimension is not at all compulsory. As in Newtonian mechanics, one can equally well regard time as a parameter. Classical particles move along time-parametrized trajectories in 3-dimensional Euclidean space, and electromagnetic fields appear as 3 -vectors labeled by time. The actual difference with Newtonian mechanics is the replacement of Galilei invariant Lagrange functions by Lorentz invariant ones. The four-dimensional Minkowskian spacetime continuum is a completely formal construct. This gets even more obvious in spacetime theories in which time is treated as imaginary quantity, e.g. in Schrödinger's interpretation of nonlinear electrodynamics [1, 2], or in the recently proposed complexification of relativistic time [3, 4].

In the general theory of relativity, the Minkowski line element is replaced by a 4-dimensional pseudo-Riemannian one. Gravitational forces manifest in the non-vanishing of the Riemannian 
curvature tensor. The connection with the principle of special relativity (Lorentz invariance) is established by the introduction of locally geodesic coordinates, so that the geodesic equations at any given point $\left(t_{0}, \mathbf{x}_{0}\right)$ reduce to $\mathrm{d}^{2} x^{\mu} / \mathrm{d} s^{2}=0$, as in Minkowski space. This is the mathematical setting for the principle of equivalence, which asserts a local Minkowskian rest frame for every 'freely falling' observer. In the presence of gravitational fields the line element can never be Minkowskian in a whole neighbourhood of $\left(t_{0}, \mathbf{x}_{0}\right)$, but Lorentz invariance is at least approximately preserved in a small locally geodesic neighbourhood. Locally, the rest frames of geodesically moving observers are still connected by (infinitesimal) Lorentz boosts. Lagrange functions and the resulting evolution equations are required to be invariant under general coordinate transformations on the 4-manifold (principle of general relativity).

This 4-dimensional world, on which our current understanding of cosmology and gravitation is based, is criticized in this article, and an approach to gravitation on the lines of the prerelativistic electromagnetism of the 18th and 19th century [5] is explored. Clearly, a theory of electromagnetism and gravitation must reproduce, in certain limits, the principle of special relativity. One of the goals of this article is to specify these limits. As for the principle of general relativity, the hypothesis of a curved 4-dimensional spacetime world cannot be regarded as established. The general theory of relativity is today, some eighty years after its appearance, still lacking far behind electrodynamics in two crucial aspects, applicability and predictive power.

The quantitative observational and experimental evidence for general relativity is primarily based on the asymptotic Schwarzschild spacetime, the general relativistic analog to Newton's gravitational potential. Cosmological solutions of Einstein's equations do not really provide quantitative predictions, e.g. for red shifts, since neither the evolution of the cosmic mass density and of the pressure, nor the global structure of the 3-space are known. In this paper, we consider only local physical processes and assume space as Euclidean, but the theory developed here can likewise be formulated in an expanding Robertson-Walker background geometry.

As for the Schwarzschild metric, we have, if we use Cartesian 3-space coordinates, the line element

$$
\mathrm{d} s^{2}=-c^{2}\left(1-\frac{2 r_{g}}{|\mathbf{x}|}+\frac{2 r_{g}^{2}}{|\mathbf{x}|^{2}}+\cdots\right) \mathrm{d} t^{2}+\left(1+\frac{2 r_{g}}{|\mathbf{x}|}+\cdots\right) \mathrm{d} \mathbf{x}^{2},
$$

with $r_{g}=k m_{s} / c^{2}$, where $k$ is Newton's gravitational constant, and $m_{s}$ is the mass of the gravitational source [6]. In eqn (1), only the orders in the asymptotic expansion that are experimentally accessible today are indicated. The coefficient $-2 r_{g}$ in the first parenthesis follows from the nonrelativistic limit (Kepler problem). The coefficient $2 r_{g}$ in the second parenthesis can be determined by the deflection of light rays by a gravitating body. If this coefficient is known, the remaining coefficient $2 r_{g}^{2}$ can be determined from planetary perihelion shifts.

In this article, we develop a Euclidean theory of gravity based on a scalar gravitational field and a permeability tensor which characterises the physical substance of space, the ether. We construct field equations by means of a phenomenological fit to the classical experiments (perihelion shift, deflection of light, and gravitational red shift). This evidently is the antithesis to the general relativistic approach to gravity, in which the field equations for the metric are derived from geometric principles. We arrive at field equations which have a much simpler structure than Einstein's, though they remain nonlinear.

Contemporary relativity principles refer to the void, the vacuum, the uniformity of space, but this kind of space is a mere geometric abstraction. To the electricians of the 18th and 19th century space meant substance and structure. Space was imagined as a medium - the ether, the carrier of electromagnetic waves. 'Light is in the ether the same thing as sound in the air', as Euler stated. At that time, it was not imaginable that waves could propagate through the void. The discovery that the speed of light is a universal constant (quantitative limits on this universality will be 
suggested in this article), and its explanation in terms of the principle of relativity, did not really disprove that physical space has substance. However, it is clear that in this context one need not contemplate on that, a purely geometric space conception turned out to be sufficient to describe electromagnetism and gravitation.

In the theory proposed here, gravitation is described by a scalar potential, like electromagnetism by a vector potential. Electromagnetic and gravitational fields propagate through the ether, which manifests by its permeability, see Sections 2 and 3. In this article we do not assume any particular micro-structure of the ether, it is macroscopically defined by a permeability tensor which affects electromagnetic and gravitational fields as well as massive particles.

Space is regarded as 3-dimensional, time as a parameter. The Euclidean space geometry is not influenced by gravitational fields, which distort only the ether. Perihelion shifts are generated by the scalar gravitational potential and the permeability tensor, see Section 4. Gravitating bodies distort the ether, which leads to a variation of its permeability; the speed of light at a given point is then anisotropic. By 'speed of light', we mean the speed of a ray or the group velocity of electromagnetic waves propagating in the ether. It must be distinguished from the vacuum speed of light, which is asymptotically defined in the homogeneous cosmic ether. Local gravitational fields diminish the speed of light; the vacuum speed remains the upper limit for the speed of signal transfer, at least as long as we do not consider particles with negative mass square (tachyons). Thus causality is preserved.

Remark. Tachyons can be incorporated in this theory of gravity, but one has to view them in the cosmic background geometry, unless one is willing to accept causality violation [7]. In [8, 9], it was argued that the expanding galactic background provides a distinguished frame of reference in which superluminal signal transfer is strictly causal. I take this opportunity to add that the concept of privileged coordinate frames in general relativity is also extensively discussed in [10].

Every mass point implies a distortion of the ether, a variation of its permeability, which destroys relativistic invariance. The concept of uniform motion and, in particular, that of observers uniformly moving in the void ceases to exist. The theory need not be Lorentz invariant, because there are no uniformly moving observers to connect by Lorentz boosts. Distortions of the ether generated by our solar system are very tiny. Kepler ellipses are entirely due to the scalar gravitational field. But the spatial variation of the ether contributes to perihelion shifts, and likewise to the deflection of light by gravitating bodies, see Section 4.

In Section 5, we define the coupling of scalar fields to the permeability tensor and the gravitational potential. We derive the semiclassical limit, the group velocity, and the kinetic energy of quantum particles propagating in the ether. The gravitational red shift is discussed, and we determine the variation of the speed of light in the gravitational potential of the earth. In Section 6 , we present our conclusions.

\section{PERMEABILITY}

The permeability of physical space is quantified by a symmetric positive definite tensor field $\gamma_{i j}^{P}(t, \mathbf{x})$; the space geometry is Euclidean. To formulate Maxwell's equations in the familiar 4tensor formalism, we formally attach a time axis to Euclidean space, and extend the Euclidean metric $\delta_{i j}$ to the Minkowski metric $\eta_{\mu v}=\operatorname{diag}(-1,1,1,1)(c=1)$, and $\gamma_{i j}^{P}$ to the 4-tensor $g_{\mu \nu}^{P}$ with components $g_{00}^{P}=-1, g_{i j}^{P}=\gamma_{i j}^{P}$ and $g_{0 m}^{P}=0$. We denote the inverse of $g_{\mu v}^{P}$ by $g^{P-1 \mu v}$, and its determinant by $g^{P}$. Greek indices run from 0 to 3, Latin ones from 1 to 3 . Indices are pulled up and down with the Minkowski metric, and on the Euclidean 3-space by $\delta_{i j}$. We define the interaction of the electromagnetic field with the permeability tensor $g_{\mu v}^{P}$, as in the case of a dielectric medium in Minkowski space [11, 12], by 


$$
S_{\mathrm{em}}=\int L_{\mathrm{em}} \mathrm{d} x \mathrm{~d} t, \quad L_{\mathrm{em}}=-\frac{1}{4} F_{\mu v} F_{\kappa \lambda} g^{P-1 \mu \kappa} g^{P-1 v \lambda},
$$

with $F_{\mu v}=A_{v, \mu}-A_{\mu, v}$. The interaction with a current is determined by $S_{\text {int }}=\int A_{\mu} j^{\mu} \mathrm{d} x \mathrm{~d} t$.

If we introduce the tensor $H^{\mu v}:=g^{P-1 \mu \kappa} g^{P-1 v \lambda} F_{\kappa \lambda}$, see e.g. [12], the field equations read

$$
H_{, \lambda}^{\kappa \lambda}=j^{\kappa}, \quad \varepsilon^{\kappa \mu \nu \lambda} F_{\mu v, \lambda}=0 .
$$

We may also write the first set of equations as

$$
\frac{1}{\sqrt{-g^{P}}} \frac{\partial\left(\sqrt{-g^{P}} H^{\mu v}\right)}{\partial x^{v}}-\left(\log \sqrt{-g^{P}}\right)_{, v} \quad H^{\mu v}=j^{\mu},
$$

the first term here being the divergence of $H^{\mu v}$ with respect to $g_{\mu v}^{P}$.

The field eqns (3) are evidently gauge invariant, and we may impose on the vector potentials the Lorentz condition $A_{v}{ }^{v}=0$ or, equally well,

$$
\frac{1}{\sqrt{-g^{P}}} \frac{\partial\left(\sqrt{-g^{P}} g^{P-1 \mu v} A_{v}\right)}{\partial x^{\mu}}=0,
$$

which is the divergence of $A_{v}$ constructed with $g_{\mu v}^{P}$. In deriving the eikonal equation from (4) $\left(j^{\mu}=0\right)$, the gauge condition (5) is preferable to the Lorentz condition. With the ansatz $A_{\mu}=\tilde{A}_{\mu} \exp (i \psi)$, one readily derives, with the usual smallness assumptions,

$$
g^{P-1 \mu v} \psi_{, \mu} \psi_{, v}=0 .
$$

We obtain the interaction of classical point particles with the permeability tensor by extending the eikonal equation to a Hamilton-Jacobi equation. Writing $S$ instead of $\psi$, we define

$$
g^{P-1 \mu v}\left(S_{, \mu}-e A_{\mu}\right)\left(S_{, v}-e A_{v}\right)=-m^{2} G^{2}(\varphi) .
$$

This is the usual Hamilton-Jacobi equation coupled to the electromagnetic 4-potentials on a manifold with metric $g_{\mu v}^{P}$. In eqn (7), we have also indicated a coupling of the mass to a scalar potential $\varphi(\mathbf{x}, t)$, which is the gravitational field in this theory. We will specify the shape of the functional $G(\varphi)$ in eqn (15). Equation (7) can be derived from the Lagrange function

$$
L=-m G(\varphi) \sqrt{-g_{\mu v}^{P} \dot{x}^{\mu} \dot{x}^{v}}+e A_{\mu} \dot{x}^{\mu} .
$$

In the limit of vanishing gravitational field, we assume that $G(\varphi) \rightarrow 1$ and $\gamma_{i j}^{P} \rightarrow \delta_{i j}$.

Remark. In a cosmological context we actually have $g_{00}^{P}=h^{2}(\tau) \rightarrow h(\tau)$ and $\gamma_{i j}^{P} \rightarrow b^{2}(\tau) \delta_{i j}$ for $\varphi \rightarrow 0$. The scale factors $h(\tau)$ and $b(\tau)$ depend on cosmic time, and are otherwise quite analogous to the expansion factor in Robertson-Walker cosmology. In local problems, if the cosmic time variation may be neglected, these factors can be scaled into the constants of nature [14, 15].

To connect with the 3-dimensional formalism of electrodynamics in a dielectric medium, one defines (see e.g. [11, 12]) 3-vectors of electric and magnetic field strengths and displacements as

$$
\begin{gathered}
E_{i}:=F_{i 0}, \quad D^{i}=H^{0 i}, \\
B^{k}:=\frac{1}{2} \varepsilon^{k i j} F_{i j}, \quad H_{i}:=\frac{1}{2} \varepsilon_{i m n} H^{m n} .
\end{gathered}
$$

Note that $E_{i}, B^{i}, D^{i}$, and $H_{i}$ are 3-vectors, whereas $F_{m n}$ and $H^{m n}$ are components of 4-tensors. We have the material relations 


$$
D^{m}=\gamma^{P-1 m n} E_{n}, \quad H_{i}=\gamma^{P-1} \gamma_{i k}^{P} B^{k},
$$

where $\gamma^{P-1 m n}$ and $\gamma^{P}$ denote the inverse and the determinant of $\gamma_{m n}^{P}$, respectively. The analogue to the electric permeability tensor is $\epsilon^{m n}:=\gamma^{P-1 m n}$, and $\mu_{i k}^{-1}:=\gamma^{P-1} \gamma_{i k}^{P}$ is the inverse of the magnetic permeability $\mu^{i k}$. In the limit $\gamma_{i j}^{P} \rightarrow \delta_{i j}$, we recover the vacuum formalism.

Now $L_{\text {em }}$ in eqn (2) may be written as

$$
L_{\mathrm{em}}=-\frac{1}{4} F_{\mu v} H^{\mu v}=\frac{1}{2}\left(E_{k} D^{k}-B^{k} H_{k}\right)
$$

and Maxwell's eqn (3) read (with $\rho:=j^{0}$ )

$$
\begin{aligned}
& B_{, l}^{l}=0, \quad \varepsilon^{k l n} E_{n, l}+\partial B^{k} / \partial t=0, \\
& D_{, l}^{l}=\rho, \quad \varepsilon^{m k i} H_{i, k}-\partial D^{m} / \partial t=j^{m} .
\end{aligned}
$$

By analogy with a dielectric medium, the energy-momentum tensor is defined as

$$
T_{v}^{\mu}:=-F_{v \alpha} H^{\mu \alpha}+\frac{1}{4} \delta_{v}^{\mu} F_{\alpha \beta} H^{\alpha \beta} .
$$

We then have as positive definite energy density $T_{0}^{0}=\frac{1}{2}\left(E_{k} D^{k}+B^{k} H_{k}\right)$.

\section{EVOLUTION EQUATIONS FOR THE SCALAR GRAVITATIONAL FIELD AND THE PERMEABILITY TENSOR}

In Section 2, we obtained the Lagrange function for the electromagnetic field and its coupling to the permeability tensor, just by replacing the inverse spacetime metric $\eta^{\mu v}$ by the inverse permeability tensor $g^{P-1 \mu v}$, see eqn (2). By this analogy, the most straightforward ansatz for the Lagrange function of the scalar gravitational field $\varphi$ is $L \sim \varphi_{, \mu} \varphi_{,} g^{P-1 \mu v}$. As a Lagrange function for the permeability tensor $g_{\mu \nu}^{P}$ one may try $L \sim \varphi g_{\kappa \lambda, \mu}^{P} g^{P-1 \kappa \lambda}{ }_{,} g^{P-1}{ }^{P}$. This is of course just a guess, and it remains to show that it works. We start with the Lagrangian

$$
\begin{aligned}
L & =-\frac{1}{2} g^{P-1 \mu v}\left(\varphi_{, \mu} \varphi_{, v}-\xi \varphi g_{\kappa \lambda, \mu}^{P} g^{P-1 \kappa \lambda}{ }_{, v}\right) \\
& =\frac{1}{2}\left(\varphi_{, t} \varphi_{, t}-\xi \varphi \gamma_{k l, t}^{P} \gamma^{P-1 k l}{ }_{, t}-\varphi_{, m} \varphi_{, n} \gamma^{P-1 m n}+\xi \varphi \gamma_{k l, m}^{P} \gamma^{P-1 k l}{ }_{, n} \gamma^{P-1 m n}\right),
\end{aligned}
$$

where $\xi$ is a dimensionless constant and $\gamma_{k l}^{P}$ denotes the Euclidean permeability tensor as defined at the beginning of Section 2. $\left(g_{\kappa \lambda}^{P}\right.$ is just a formal extension of $\left.\gamma_{k l}^{P}\right)$. In eqns (7) and (8), we choose

$$
G(\varphi)=\varphi^{-1 / 2} \tanh \varphi^{1 / 2}=1-\frac{1}{3} \varphi+\frac{2}{15} \varphi^{2}-\cdots,
$$

which defines the interaction of $\varphi$ with massive particles; $\varphi$ must be positive. We do not have a derivation of the Lagrangian (14) and the coupling (15) from 'first principles', but they lead to the right experimental results, and to a self-consistent theory; also see Remark 4 at the end of this section.

From eqn (14), we obtain the field equations for the gravitational potential $\varphi(t, \mathbf{x})$ and the permeability tensor $\gamma_{m n}^{P}(t, \mathbf{x})$ in Cartesian coordinates as 


$$
\begin{gathered}
-\varphi_{, t, t}+\gamma^{P-1 k l} \varphi_{, k, l}+\gamma^{P-1 k l}{ }_{, l} \varphi_{, k}-\frac{1}{2} \xi \gamma_{k, t,}^{P} \gamma^{P-1 k l}{ }_{,}+\frac{1}{2} \xi \gamma_{i j, k}^{P} \gamma^{P-1 i j}{ }_{,} \gamma^{P-1 k l}=0, \\
-\gamma_{m n, t, t}^{P}+\gamma_{i m, t}^{P} \gamma_{j n, t}^{P} \gamma^{P-1 i j}-\varphi^{-1} \varphi_{, t} \gamma_{m n, t}^{P}+\gamma_{m n, i, j}^{P} \gamma^{P-1 i j}+\gamma_{m n, i}^{P} \gamma^{P-1 i j}{ }_{j}-\frac{1}{2} \gamma_{i j, m}^{P} \gamma^{P-1 i j}{ }_{, n} \\
-\gamma_{m, k}^{P} \gamma_{n j, i}^{P} \gamma^{P-1 i j} \gamma^{P-1 k l}+\varphi^{-1} \varphi_{, i} \gamma_{m n, j}^{P} \gamma^{P-1 i j}+\frac{1}{2} \xi^{-1} \varphi^{-1} \varphi_{, m} \varphi_{, n}=0 .
\end{gathered}
$$

Here time is a parameter, not a dimension; $\varphi$ and $\gamma_{m n}^{P}$ are fields defined in Euclidean space with metric $\delta_{m n}$.

Next we calculate the analogue to the general relativistic Schwarzschild solution, namely the static, spherically symmetric potential of a point source of mass $m_{s}$. The field equations cannot be exactly solved, but it is very easy to obtain an asymptotic expansion. We start with the ansatz

$$
\begin{gathered}
\varphi(r)=\alpha_{1} / r+\alpha_{2} / r^{2}+O\left(1 / r^{3}\right), \\
\gamma_{i j}^{P}=(1+b(r)) \delta_{i j}+c(r) x_{i} x_{j} / r^{2}, \\
b(r)=\beta / r+O\left(1 / r^{2}\right), \quad c(r)=\gamma / r+O\left(1 / r^{2}\right) .
\end{gathered}
$$

The orders indicated are sufficient to compare with available experimental results. One can insert this ansatz into the field equations and determine the relations between the coefficients $\alpha_{i}, \beta$ and $\gamma$ by comparing powers of $1 / r$. Another possibility is to insert eqn (19) into eqn (14), and to vary the functions $\varphi(r), b(r)$ and $c(r)$. To the accuracy indicated in eqns (18) and (20), we may then use the Lagrangian

$$
L(\varphi, b, c)=-\frac{1}{2} r^{2}\left(\varphi^{\prime 2}(1-b(r)-c(r))+\xi \varphi\left(3 b^{\prime 2}+c^{\prime 2}+4 r^{-2} c^{2}(r)+2 b^{\prime} c^{\prime}\right)\right),
$$

the $r^{2}$ stemming from the volume element in the action.

From the field eqn (16) and eqn (17) or the Euler equations derived from (21), we obtain three relations among the coefficients $\alpha_{i}, \beta$ and $\gamma$, namely

$$
\gamma=\frac{\alpha_{1}}{10 \xi}, \beta=-\frac{\alpha_{1}}{5 \xi}, \alpha_{2}=\frac{-7}{400 \xi} \alpha_{1}^{2} .
$$

We regard $\alpha_{1}$ as integration constant and write $\alpha_{1}=3 r_{g} ; r_{g}$ will be identified as the gravitational radius of the source. With eqn (18), eqn (20) and eqn (22), we obtain

$$
\begin{gathered}
G(\varphi)=1-\frac{r_{g}}{r}+\frac{r_{g}^{2}}{r^{2}}\left(\frac{21}{400 \xi}+\frac{6}{5}\right)+O\left(\left(r_{g} / r\right)^{3}\right), \\
\gamma_{i j}^{P}=\left(1-\frac{3}{5 \xi} \frac{r_{g}}{r}\right) \delta_{i j}+\frac{3}{10 \xi} \frac{r_{g}}{r} \frac{x_{i} x_{j}}{r^{2}}+O\left(\left(r_{g} / r\right)^{2}\right) .
\end{gathered}
$$

If we use these expressions in the Lagrangian (8), we recover for $r_{g}=m_{s} k / c^{2}$ the Newtonian limit, and for $\xi=-3 / 40$ the Einsteinian results for the deflection of light and perihelion shifts, see Section 4.

Remark 1. To obtain the potentials in the region $r \approx r_{g}$, the exact field eqns (16) and (17) have to be integrated with the asymptotic solution (18) to (20) and (22) as boundary condition. I expect that a singularity will appear in this region; for example, $\gamma_{i j}^{P}$ may cease to be positive definite. This singularity would then define a finite particle radius. Unlike the case of general relativity, the singularity cannot be removed by coordinate transformations, for the underlying space is 
invariably Euclidean. This radius could decisively change the self-energy of a classical point particle. One would then integrate the energy density (derived from eqn (14) via standard Lagrange formalism) only up to this radius, and so possibly obtain a finite self-energy. There are of course no spacetime singularities in this theory.

Remark 2. The Lagrangian (14) does not permit a proper linearization. The reason for this is that the gravitational field $\varphi$ approaches far away from sources zero, not a finite constant. So linearization means to drop the $\xi$-term in eqn (14) as a whole, namely the Lagrangian for the permeability tensor $\gamma_{m n}^{P}$. In the wave eqn (16) for the $\varphi$-potential, the $\xi$-terms can be neglected if the permeability tensor is weakly varying in space and time, but the wave eqn (17) for the permeability tensor is inextricably coupled to the $\varphi$-field. There is no transversality condition on $\gamma_{m n}^{P}$. The only conditions imposed are that $\varphi$ is positive and $\gamma_{m n}^{P}$ is positive definite. The field eqn (16) and (17) determine the fields completely, once that initial and boundary conditions are set; there are no arbitrary functions (gauge freedom) in the solutions.

Remark 3. In the limit $\gamma_{m n}^{P} \rightarrow \delta_{m n}$, we obtain from eqn (14) the Lagrangian of a massless scalar field theory in Minkowski space. But as a rule, massive particles generate a non-trivial permeability tensor in their vicinity, and so relativistic invariance can under no circumstances be strictly obtained. Because a permeability tensor differing from the Euclidean metric always generates acceleration, non-accelerated observers are an idealization which is in principle unattainable.

Remark 4. My initial motivation for Lagrangian (14) was taken from [13], where Lagrangians for linear gravitational waves are constructed (in a general relativistic cosmology), which are consistent with the gauge conditions usually imposed on linear gravitational waves. Because of the gauge condition $g_{0 n}=0$ on the spacetime metric, it is tempting to substitute for $g_{00}$ a scalar field $\varphi$ containing the Newtonian potential, and to regard the 3-metric $g_{m n}$ as permeability tensor in Euclidean space. Lagrangian (14) is much simpler structured than the Lagrangians considered in [13]; it seems to be the simplest choice to be found that leads to Euler equations which determine the fields uniquely (see Remark 2), and gives rise to the right perihelion shift. If one starts with Lagrangian (14), then the indicated terms of the power series expansion in eqn (15) are essential to match the experimental results for perihelion shifts and deflection angles. The $\tanh \varphi^{1 / 2}$ in eqn (15) is an extrapolation based on these terms.

Remark 5. In eqn (14), we did not include source terms, e.g. the Lagrangians (2) or (35). In this paper, we study the potential of a static point mass $m_{s}$. The gravitational constant as well as $m_{s}$ enter in the solution of eqns (16) and (17) via asymptotic boundary conditions; the potential $\varphi$ must produce Kepler ellipses, see eqn (32).

\section{CLASSICAL PARTICLES IN THE ETHER}

In Section 2, we have already introduced the Lagrange function for classical point particles interacting with the scalar gravitational potential and the permeability tensor. If we choose the curve parameter $s$ so that $G^{2}(\varphi) g_{\mu v}^{P} \dot{x}^{\mu} \dot{x}^{v}=-1$, we readily obtain from eqn (8) the equations of motion

$$
G^{2}(\varphi)\left(g_{\alpha \mu}^{P} \ddot{x}^{\mu}+g_{\alpha \mu, v}^{P} \dot{x}^{\mu} \dot{x}^{v}-\frac{1}{2} g_{\mu v, \alpha}^{P} \dot{x}^{\mu} \dot{x}^{\nu}\right)+2 G G_{, v} \dot{x}^{v} g_{\alpha \mu}^{P} \dot{x}^{\mu}-\frac{e}{m} F_{\alpha \beta} \dot{x}^{\beta}+G^{-1} G_{, \alpha}=0
$$

Introducing time as curve parameter, 


$$
d s=G(\varphi) \sqrt{1-\gamma_{i j}^{P} x^{i} x^{\prime j}} \mathrm{~d} t,
$$

where $x^{i}:=\mathrm{d} x^{i} / \mathrm{d} t$, we may write the action leading to eqn (25) in 3-dimensional form, $S=\int L(t) \mathrm{d} t$, with

$$
L(t)=-m G(\varphi) \sqrt{1-\gamma_{i j}^{P} x^{\prime i} x^{\prime j}}+e A_{0}+e A_{i} x^{\prime i} .
$$

If the fields $\varphi$ and $\gamma_{i j}^{P}$ are time independent, and in the absence of electromagnetic forces, we obtain from the $\alpha=0$ component of the Lagrange eqn (25) the conservation law $\mathrm{d} E / \mathrm{d} t=0$, where

$$
E=\frac{m G(\varphi)}{\sqrt{1-\gamma_{i j}^{P} x^{\prime i} x^{\prime j}}}
$$

The kinetic energy in the ether we extract from the total energy as $E_{\text {kin }}=E G^{-1}(\varphi)$.

From the eikonal eqn (6), we have for light rays $\gamma_{i j}^{P} x^{\prime i} x^{\prime j}=1$. The speed of light in the direction of a Euclidean unit vector $e^{i}$ is accordingly $\left|\mathbf{v}_{c}\right|=\left(\gamma_{i j}^{P} e^{i} e^{j}\right)^{-1 / 2}$. Let us consider a particle moving with speed $x^{\prime i}=|\mathbf{v}| e^{i}$. We then have $\gamma_{i j}^{P} x^{\prime i} x^{\prime j}=|\mathbf{v}|^{2} /\left|\mathbf{v}_{c}\right|^{2}$, with $\mathbf{v}_{c}$ the speed of light in the direction of $\mathbf{v}$. So the roots $\sqrt{1-\gamma_{i j}^{P} x^{\prime i} x^{\prime j}}$ in the preceding equations can be replaced by $\sqrt{1-|\mathbf{v}|^{2} /\left|\mathbf{v}_{c}\right|^{2}}$.

Perihelion shifts are calculated from the asymptotic solutions (23) and (24). This amounts to solving the Kepler problem in the metric $\hat{g}_{\mu v}:=G^{2} g_{\mu v}^{P}$, see eqn (7). Equation (24) gives $\gamma_{i j}^{P}$ in Cartesian coordinates. In polar coordinates $r, \psi, \theta=0$, we have

$$
\hat{g}_{\mu v} \mathrm{~d} x^{\mu} \mathrm{d} x^{v}=-c^{2}\left(1+\frac{\tilde{\alpha}}{r}+\frac{\tilde{\beta}}{r^{2}}+O\left(r^{-3}\right)\right) \mathrm{d} t^{2}+\left(1+\frac{\tilde{\gamma}}{r}+O\left(r^{-2}\right)\right) \mathrm{d} r^{2}+r^{2}\left(1+O\left(r^{-1}\right)\right) \mathrm{d} \psi^{2},
$$

where

$$
\tilde{\alpha}:=-2 r_{g}, \quad \tilde{\beta}:=\left(\frac{21}{200 \xi}+\frac{17}{5}\right) r_{g}^{2}, \tilde{\gamma}:=\left(-\frac{3}{10 \xi}-2\right) r_{g} .
$$

We have indicated here only the orders needed to calculate the perihelion shift and the deflection of light, and we depart from now on from the convention $c=1$. (Note that $r_{g}$ is an integration constant, see eqn (22), and $\xi$ is the dimensionless constant in the Lagrangian (14).) The HamiltonJacobi equation reads as

$$
\hat{g}^{-1 t t}\left(\frac{\partial S}{\partial t}\right)^{2}+\hat{g}^{-1 r r}\left(\frac{\partial S}{\partial r}\right)^{2}+\hat{g}^{-1 \psi \psi}\left(\frac{\partial S}{\partial \psi}\right)^{2}+m^{2} c^{2}=0
$$

where $\hat{g}^{-1 t t}, \hat{g}^{-1 r r}$ and $\hat{g}^{-1 \psi \psi}$ are the respective diagonal components of the inverse of $\hat{g}_{\mu v}$. With the ansatz $S=\left(-m c^{2}+E\right) t+M \psi+S(r)$, we obtain from eqns (29) and (31)

$$
\begin{aligned}
S(r)=\int \mathrm{d} r\left[2 m E\left(1+\frac{1}{2} \frac{E}{m c^{2}}\right)-\right. & \frac{\tilde{\alpha}}{r} m^{2} c^{2}\left(1+\frac{\tilde{\alpha}-\tilde{\gamma}}{\tilde{\alpha}}\left(\frac{2 E}{m c^{2}}+\frac{E^{2}}{m^{2} c^{4}}\right)\right) \\
& \left.+\frac{1}{r^{2}}\left(m^{2} c^{2}\left(\tilde{\alpha}^{2}-\tilde{\beta}-\tilde{\alpha} \tilde{\gamma}\right)\left(1+\frac{2 E}{m c^{2}}+\frac{E^{2}}{m^{2} c^{4}}\right)-M^{2}\right)+O\left(r^{-3}\right)\right]^{1 / 2}
\end{aligned}
$$

The Kepler ellipses are recovered if we put $r_{g}=m_{s} k / c^{2}$ in eqns (30). From eqns (32) and (30), we can read off the perihelion shift [6] 


$$
(\Delta \psi)_{\text {perihel }}=\frac{\tilde{\alpha}^{2}-\tilde{\beta}-\tilde{\alpha} \tilde{\gamma}}{r_{g}^{2}} \frac{\pi k m_{s}}{c^{2} a\left(1-e^{2}\right)}, \quad \frac{\tilde{\alpha}^{2}-\tilde{\beta}-\tilde{\alpha} \tilde{\gamma}}{r_{g}^{2}}=-\frac{141}{200 \xi}-\frac{17}{5},
$$

where $a$ is the length of the major semiaxis, and $e$ the eccentricity. For the deflection angle of light, one obtains from eqn (32) (with $m=0$ ) and eqn (30)

$$
(\Delta \psi)_{\text {light }}=\frac{\tilde{\gamma}-\tilde{\alpha}}{r_{g}} \frac{k m_{s}}{c^{2} \rho}, \frac{\tilde{\gamma}-\tilde{\alpha}}{r_{g}}=-2-\frac{3}{10 \xi},
$$

where $\rho$ is the shortest distance of the bent ray to the center of the source $m_{s}$. With $\xi=-3 / 40$, we recover the general relativistic results for perihelion shift and deflection angle. A value of $\xi=-1 /(13.3 \pm 0.1)$ is in accordance with observations.

\section{ETHER AND WAVE MECHANICS}

From the Hamilton-Jacobi eqn (7), it is clear how to choose the Lagrange function for the corresponding scalar quantum field $\left(c=\hbar=1, A_{\mu}=0\right)$,

$$
L=-\frac{1}{2}\left(g^{P-1 \mu v} \psi_{, \mu} \overline{\psi_{, v}}+G^{2}(\varphi) m^{2} \psi \bar{\psi}\right)
$$

The wave equation derived from eqn (35) reads

$$
\left(\partial_{\mu} g^{P-1 \mu v} \partial_{v}-G^{2}(\varphi) m^{2}\right) \psi=0 .
$$

We study this equation in the central potentials (23), (24). For spherically symmetric wave solutions we use in eqn (36) the ansatz $\psi=\exp (-i \omega t) \hat{\psi}(r)$, and so obtain, to the accuracy indicated in eqn (20),

$$
\left[\frac{1}{r^{2}}\left(1-\frac{3}{2} b(r)-\frac{1}{2} c(r)\right) \frac{\mathrm{d}}{\mathrm{d} r}\left(r^{2}\left(1+\frac{1}{2} b(r)-\frac{1}{2} c(r)\right) \frac{\mathrm{d}}{\mathrm{d} r}\right)+\omega^{2}-m^{2} G^{2}(\varphi)\right] \hat{\psi}=0 .
$$

With eqns (20), (22) and (23), we have asymptotically

$$
\begin{gathered}
\psi \sim \frac{1}{r} \exp \left(i\left(\int \tilde{k}(r) \mathrm{d} r-\omega t\right)\right), \omega:=\sqrt{k^{2}+m^{2}}, \\
\tilde{k}(r):=k\left(1+\left(\frac{m^{2}}{k^{2}}-\frac{3}{20 \xi}\right) \frac{r_{g}}{r}\right)+O\left(r_{g}^{2} / r^{2}\right) .
\end{gathered}
$$

The $m^{2}$ term in $\tilde{k}(r)$ stems from $G^{2}(\varphi)$, the $\xi$ term from $b(r)$ and $c(r)$ in $\gamma_{i j}^{P}$; $k$ is the usual spectral parameter. For the radial group velocity, we readily obtain

$$
v_{\mathrm{gr}}=\frac{k}{\sqrt{k^{2}+m^{2}}}\left(1+\left(\frac{m^{2}}{k^{2}}+\frac{3}{20 \xi}\right) \frac{r_{g}}{r}\right)+O\left(r_{g}^{2} / r^{2}\right)
$$

The corresponding classical problem of radial motion in the potential (23), (24) is defined, see eqn (8), by the Lagrangian

$$
L^{2}=G^{2}(\varphi)\left(\dot{t}^{2}-(1+b(r)+c(r)) \dot{r}^{2}\right) .
$$

Integrals of motion are $L^{2}=1$ and $m G^{2}(\varphi) \dot{t}=E$. The integration constant $E$ is just the total energy as defined in eqn (28). The radial velocity reads 


$$
v=|\dot{r} / \dot{t}|=(1+b(r)+c(r))^{-1 / 2}\left(1-m^{2} E^{-2} G^{2}(\varphi)\right)^{1 / 2} .
$$

If we identify the parameters $k$ and $E$ as $E=\sqrt{k^{2}+m^{2}}$, we see that $v$ coincides, for $k \rightarrow \infty$, with the group velocity (39). If we drop the mass terms in eqns (39) and (41), we obtain the speed of light in the ether,

$$
v_{c}=\frac{1}{\sqrt{1+b(r)+c(r)}}=1+\frac{3}{20 \xi} \frac{r_{g}}{r}+O\left(r_{g}^{2} / r^{2}\right)
$$

Remark 1. To restore the natural units in eqns (38), (39) and (42), we replace in these formulas $m$ by $m c / \hbar$. Moreover, the right-hand side of the equations for $\omega, v$, and $v_{c}$ must be multiplied by $c$, and $E=\hbar \omega$.

Remark 2. In the limit $r \rightarrow \infty$, the vacuum speed $c$ is recovered in eqn (42), see Remark 3 at the end of Section 3. Because $\xi$ is negative, the permeability generated by a static point source diminishes the speed of light below the vacuum speed.

In the semiclassical approximation, we have as kinetic energy of a particle/photon, see eqns (28) and (23),

$$
E_{\mathrm{kin}}=\hbar \omega G^{-1}(\varphi) \approx \hbar \omega\left(1+r_{g} / r\right) .
$$

This scaling of the photon energy by the factor $\left(1+r_{g} / r\right)$ can be measured by Mössbauer resonance absorption. Note that the frequency $\omega$ is independent of $r_{g} / r$, what scales is the kinetic energy of the photon. For the wave length we have of course $\lambda=1 / \tilde{k}(r)$, and for the phase velocity $v_{\mathrm{ph}}=\lambda \omega$, see eqn (38). Note that this scaling is very different from the general relativistic gravitational red shift, where $\lambda \sim\left(1-\mathrm{r}_{g} / r\right), \omega \sim\left(1+r_{g} / r\right)$, and $v_{\mathrm{ph}}=c$ does not scale at all.

Let us finally return to eqn (42). The speed of light scales due to the permeability of the ether by a factor $1+3 r_{g} /(20 \xi r)$. This is the most important prediction. We denote by $v_{c}(r)$ the speed of light at distance $r$ from the source. With $r=R_{\oplus}, m_{s}=M_{\oplus}$, and $\xi=-3 / 40$, we obtain $c / v_{c}\left(R_{\oplus}\right)-1=1.4 \times 10^{-9}$. More generally, $v_{c}\left(\alpha R_{\oplus}\right) / v_{c}\left(R_{\oplus}\right)-1=2\left(1-\alpha^{-1}\right) r_{g \oplus} / R_{\oplus}$, for $\alpha>1$, and $r_{g \oplus} / R_{\oplus}=6.9 \times 10^{-10}$. This relation could be checked by a satellite experiment.

\section{CONCLUSION}

"Outside of pure mathematics, anyone who pronounces the word 'impossible' is lacking in prudence", Arago once claimed. In the theory presented here, the basic principles of general relativity are questioned: the principle of general covariance, the equivalence principle, and the universality of the speed of light. This ether theory is not derived from and based on geometric principles; it is phenomenological, designed in a way to fit the fundamental experiments on gravitation.

In gravitational fields the speed of light is varying due to distortions of the ether. Gravitational fields always generate distortions and so prevent uniform motion, though the deviations from uniform motion are very small if generated by fields presently accessible to us. The notion of a freely falling observer is approximate, but the equivalence of gravitating and inertial mass holds, since in the absence of electromagnetic fields the mass drops out in the equations of motion. Sufficiently far away from gravitational sources, Lorentz boosts can be defined by means of the speed of light in the homogeneous cosmic ether. The universality of the speed of light and the equivalence principle are recovered in this limit, but otherwise these principles are only idealizations based on a purely geometric spacetime view. 
The cosmological consequences of this ether theory, in particular the impact of the ether on the cosmological red-shift and on the variation of the constants of nature in cosmic time are studied in $[14,15]$. Research on cosmic chaos as outlined in [7] is further pursued in [16, 17].

Acknowledgements - The author acknowledges the support of the Japan Society for the Promotion of Science, contract No. P-96409. Inspiring stays at the Inter-University Centre for Astronomy and Astrophysics, Pune, the Institute of Physics, Bhubaneswar, and the Centre for Theoretical Studies, Indian Institute of Science, Bangalore, are gratefully acknowledged. In particular, I would like to thank Prof. C.V. Vishveshwara for initiating my visit to the IIAP, and Prof. V. Krishan for her constant encouragement.

\section{REFERENCES}

1. Schrödinger, E., Born's new field theory. Proc. Roy. Soc. London Ser. A, 1935, 150, 465-477.

2. Hoffmann, B., Gravitational and electromagnetic mass in the Born-Infeld electrodynamics. Phys. Rev., 1935, 47, $877-880$.

3. El Naschie, M. S., On conjugate complex time and information in relativistic quantum theory. Chaos, Solitons and Fractals, 1995, 5, 1551-1555.

4. Argyris, J. and Ciubotariu, C., On El Naschie's complex time and gravitation. Chaos, Solitons and Fractals, 1997, 8, $743-751$.

5. Whittaker, E., A History of the Theories of Aether and Electricity, Vol. 1. Thomas Nelson and Sons, London, 1951.

6. Landau, L. D. and Lifshitz, E. M., The Classical Theory of Fields. Pergamon, London, 1971.

7. Tomaschitz, R., Tachyonic chaos and causality in the open universe. Chaos, Solitons and Fractals, $1996,7,753-768$.

8. Tomaschitz, R., Tachyons in an expanding space-time. Chaos, Solitons and Fractals, 1997, 8, 761-766.

9. Tomaschitz, R., Tachyons in Robertson-Walker cosmology. Intern. J. Mod. Phys. D, 1998, 7, $279-298$.

10. Fock, V. A., The Theory of Space, Time, and Gravitation. Pergamon, New York, 1959.

11. Panofsky, W. and Phillips, M., Classical Electricity and Magnetism. Addison-Wesley, Reading, Mass, 1962.

12. Volkov, A. M. and Kiselev, V. A., Proper frequencies of a rotating ring resonator. Sov. Phys. JETP, 1970, 30, 733736.

13. Tomaschitz, R., Gravitational waves and electrodynamic formalism. Internat. J. Theoret. Phys., 1997, 36, $955-977$.

14. Tomaschitz, R., Cosmic ether. Intern. J. Theoret. Phys., 1998, 37, 1121-1140.

15. Tomaschitz, R., How rigid are galactic measuring rods? (in preparation).

16. Tomaschitz, R., Chaos in the galactic dynamics. Fractals, 1997, 5, 215-220.

17. Tomaschitz, R., Chaos and topological evolution in cosmology. Intern. J. Bifur. Chaos, 1997, 7, $1847-1853$. 